\title{
Editorial for the Special Issue on Manufacturing Informatics for Intelligent Automation
}

Proceedings of the IMechE Part B, Journal of Engineering Manufacture

\author{
Guest Editors: Professor Ashutosh Tiwari, Professor Phil Webb and Dr Vinayak Prabhu
}

Informatics and automation play a vital role in today's complex, constantly evolving and knowledge-intensive manufacturing systems. Automation brings increased levels of standardisation to such systems and has the potential to enable long term consistency in process reliability, product quality and manufacturing productivity. Informatics enables manufacturing enterprises to understand and digitise their operations and as a result enhance product and service quality, reduce waste, increase efficiency and reduce the time to market. Successful adoption of informatics to develop intelligent automation solutions is enabled by recent advances in, and widespread use of, Information and Communications Technology (ICT).

Despite the ever increasing use of automation in the global manufacturing industry, there are still many manufacturing operations that are not fully automated. Primary reasons for this are the level of task complexity, lack of complete understanding of the task and unfavourable cost-to-benefit ratios of adopting automation. Therefore, there is a need to look for new, innovative and cost effective solutions to understand and digitise complex manufacturing operations and develop intelligent automation solutions for them that incorporate flexibility and adaptability in this constantly evolving manufacturing sector. This special issue is a collection of state-of-the-art research papers that report some possible solutions by leveraging the latest scientific and technological developments in informatics and ICT.

The special issue received novel and impactful contributions from researchers and practitioners, who are developing innovative solutions in the area of manufacturing informatics for intelligent automation. The selected papers reflect the latest research and industrial best practices in the areas of digitisation of manufacturing operations, capture and modelling of human task data, real-time monitoring and analysis of manufacturing systems, use of machine learning for characterisation, prediction, optimisation and management of production systems, intelligent decision-making, and adaptive and reconfigurable manufacturing systems.

The first paper titled "A Dual Scheduling Model for Optimizing Robustness and Energy Consumption in Manufacturing Systems" presents a knowledge-based model to analyse previous incidences of process disruptions in a manufacturing system, solved by a memetic algorithm which combines a genetic algorithm (GA) with a local search (LS) method to obtain robust and energy-aware scheduling solutions. The second paper titled "Modelling, Monitoring and Evaluation to Support Automatic Engineering Process Management" investigates techniques, such as autonomic computing, data mining and knowledge management to support the automation of engineering process management from a datadriven perspective. The third paper titled "A Strategy to Analyse a Composite Design to Improve Automated Production Speeds" reports methodologies for indicating the likely impact of composite design on automated manufacture at the design optimisation stage and presents recommendations for using these methodologies for improving composite deposition speeds. The fourth paper titled "Adaptive Robust Control of Machining Force and Contour Error with Tool Deflection Using Global Task Coordinate Frame” proposes an adaptive and robust machine controller that maintains high performance control of both force 
and contour error in the presence of significant model errors or external disturbances. The fifth paper titled "Decision Support System Enabled by Depth Imaging Sensor Data for Intelligent Automation of Moving Assemblies" demonstrates that a human-like decision making system can be developed by using expert rules derived from heuristics and real-time operation data collected from the shopfloor by consumer-grade gaming sensors. The sixth paper titled "Production Control Strategy Inspired by Neuroendocrine Regulation" presents an adaptive control model of production system, inherited from the hormone regulation principle, that is integrated with a backlog controller and a work-in-progress (WIP) controller in order to reduce backlog variation and keep a defined WIP level at all times. The seventh paper titled "Characterization of Wear and Prediction of Wear Zone Locations on the Rake Face Using Mamdani Fuzzy Inference System" proposes a method to identify, characterise and locate the actual wear regions on the chip contact area of Tungsten Carbide (WC) cutting inserts, which will enable localised nano-coatings rather than total rake face coating thereby reducing the cost of production. The eighth paper titled "A Study to Trial the Use of Inertial Non-Optical Motion Capture for Ergonomic Analysis of Manufacturing Work" describes a case study conducted within the aerospace manufacturing industry where data on human activities involved in aircraft wing system installations captured using automatic non-optical motion capture system were found to be more accurate and suitable for digital modelling than traditional ethnographic methods. Finally, the ninth paper titled "A Hormone Regulation Based Approach for Distributed and On-Line Scheduling of Machines and AGVs" reports a hormone regulation based approach for online scheduling of machines and AGVs within a distributed system that assigns emergent tasks and generates feasible schedules with improved scheduling quality as compared to benchmarks in literature. The above nine papers encompass a broad range of novel and advanced informatics techniques that enable the intelligent automation of manufacturing tasks such as process design, scheduling, monitoring and control, including the human-related aspects of real-time decision making and ergonomic analysis. 
2017-01-01

\title{
Editorial for the special issue on
} manufacturing informatics for intelligent automatior

\author{
Tiwari, Ashutosh
}

SAGE Publications (UK and US)

Tiwari A, Webb P, Prabhu V, Editorial for the special issue on manufacturing informatics for intelligent automation, Proceedings of the Institution of Mechanical Engineers, Part B: Journal of Engineering Manufacture, online 1 January 2017, DOI: 10.1177/0954405417694063.

http://dx.doi.org/10.1177/0954405417694063

Downloaded from Cranfield Library Services E-Repository 